\title{
La innovación educativa y la vinculación con talleres participativos turísticos en la provincia de los Ríos
}

\author{
DOI: $\underline{\text { https://doi.org/10.33262/ap.v3i4.134 }}$

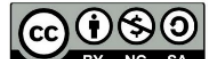

Educational innovation and linkage with participatory tourism workshops in the province of Los Rios

Christian Rivera García. ${ }^{1}$, Darío Arellano Valencia. ${ }^{2} \&$ Xiomara Noemi Reyes Indio. ${ }^{3}$

\begin{abstract}
Introduction. Educational innovation contemplates several constructive areas: technology, didactics, pedagogy, processes and people, integrated to transform the teaching-learning process, maintaining the novelty of social contexts, preserving the collective millennial memory in the integration and inclusion of the different educational actors and recreational activities at the national level where the Technical University of Babahoyo through its program of outreach projects is forged socially to lead and achieve safeguards in solving problems, making decisions, generating substantial knowledge on the relevance of university careers incorporates experience in substantive, multidisciplinary, multilevel theory and practice, human and professional contextual transformation through participatory workshops collaborate with synchronous and asynchronous virtual learning providing updated information with multiple direct benefits for development projects or community. Target. Innovate in a series of free participatory tourist workshops to support the linkage of the population towards new alternatives for local employability. Methodology. This research had two stages, one directed towards updating the baseline of the region, conspiring with institutional research lines, and the second stage includes the design of the information collection by applying a digital survey to the participants. Conclusions. The results of the survey carried out with 100 people owe that the need for post-pandemic economic reactivation lies in the safety of applying
\end{abstract}

\footnotetext{
1 Universidad Técnica de Babahoyo FCJSE, Ecuador. crivera@utb.edu.ec

2 Universidad Técnica de Babahoyo FCJSE, Ecuador. darellano@utb.edu.ec

3 Universidad Técnica de Babahoyo FCJSE, Ecuador. xiomi140601@gmail.com
} 
the vaccine under all national and international risks, seeking strategic allies and investors that promote the financing of new tourism goods and services.

Keywords: educational innovation, didactics, linking projects, multidisciplinary, baseline.

\section{Resumen}

Introducción. La innovación educativa contempla varias áreas constructivas: tecnología, didáctica, pedagogía, procesos y personas, integradas para transformar el proceso de enseñanza-aprendizaje, manteniendo la novedad de contextos sociales, conservando la memoria milenaria colectiva en la integración e inclusión de los diferentes actores educativos y recreativos en el ámbito nacional donde la Universidad Técnica de Babahoyo mediante su programa de proyectos de vinculación se forja socialmente para conducir y lograr la salvaguarda en la solución de problemas, la toma de decisiones, generando conocimientos sustanciosos en la pertinencia de las carreras universitarias incorpora experiencia en la teoría y práctica sustantiva, multidisciplinar, multiniveles, transformación contextual humana y profesional a través de los talleres participativos colaboran con el aprendizaje virtual sincrónico y asincrónico brindando información actualizada con múltiples beneficios directos para los proyectos de desarrollo comunitario. Objetivo. Innovar en una serie de talleres participativos turísticos gratuitos para apoyar la vinculación de la población hacia nuevas alternativas de empleabilidad local. Metodología. Esta investigación tuvo dos etapas una direccionada hacia la actualización de la línea base de la región conspirando con líneas de investigación institucionales y la segunda etapa incluye el diseño de la recolección de la información aplicando una encuesta digital a los participantes. Conclusiones. Los resultados de la encuesta realizada a 100 personas debelo que las necesidades de reactivación económica post pandemia recae en la seguridad de aplicarse la vacuna bajo todo riesgo nacional e internacional, buscar aliados estratégicos e inversionistas que propicien el financiamiento de nuevos bienes y servicios turísticos.

Palabras Clave: innovación educativa, didáctica, proyectos de vinculación, multidisciplinar, línea base.

\section{Introducción}

Los escenarios urbanos y rurales, públicos y privados, se encuentran resquebrajados por el golpe global de pandemia, estos requieren de tratos diferentes cuando incorporamos actividades de desarrollo turístico, reunir elementos claves en estos procesos que demanda diseños especiales de integración, mediante la investigación acción poblacional. En las ciudades de Babahoyo, Ventanas y Quinsaloma, los participantes recrudecen su memoria milenaria colectiva al encontrarse motivados en respuesta a la innovación en el contexto de trabajo direccionado en particular hacia las mujeres y jóvenes de la ciudad y sus parroquias rurales, mediante talleres participativos con presupuesto valorativo comprendido en la cosmovisión que une generaciones hereditarias de los recursos naturales y culturales. La comunidad fortalece la participación y la puede extender con la 
asociatividad de sus integrantes y también incluye el rescate de la identidad puesta en marcha cuando intercambiamos experiencias en convivencia participativa, teniendo como sujeción turística de este entramado la innovación educativa.

El primer supuesto para la innovación educativa destaca su carácter sistémico, como Havelock \& Huberman (1977), señalaron en su obra sobre la innovación educativa en los países en desarrollo, la teoría de sistemas es un marco adecuado para los proyectos de innovación educativa.

Esta base considera la innovación educativa desde un enfoque de responsabilidad universitaria argumentados en los siguientes autores: Epper et al. (2004), Salinas (2004), Palomo et al. (2006), De Pablos et al. (2007b), De Pablos et al. (2007a), García \& Valcárcel (2007) y García et al. (2012), el rigor de estos expertos está circunscrita a la relación de la innovación con las experiencias universitarias en manifestaciones de solución de problemas, previo a la formación de mentes reflexivas, con pensamientos libres de prejuicios sociales y comerciales, ejemplados con raciocinio ético y moral del claustro docente, autoridades, administrativos y empleados.

La innovación educativa contiene un marco direccional como constante fija de un contexto volátil de objetividad con ideas prácticas, materiales, contenidos nuevos, metodologías aplicativas, patrones de respuesta cultural, interrelación entre personal y normas establecidas en la sociedad civil, situaciones educativas originales que han reemplazado la normalidad interiorizada en las instituciones educativas como norma imperecedera de cambio, conspirando con: lo nuevo ya es ordinario, la razón que los mueve son las necesidades insatisfechas y al mismo tiempo las exigencias académicas. Las reformas educativas siguen la respuesta a una objetividad preclara de soluciones directas, estos cambios son estructurales, en subsistemas eslabonados que tratan de fragmentar estereotipos educativos internacionales resistentes que dificultan la intencionalidad viable, pero confusa por la misma cultura organizacional que resiste a sus hábitos extremos automatizados e ineficaces. Los paradigmas educativos impregnados de innovación son la esencia de objetivos institucionales bien planificados y sujetos a verificación constante. Las unidades académicas universitarias integran planificaciones que reflejan un cronograma ejecutado con sistemas de evaluación real y permanente, evidenciando problemas y soluciones futuristas con pensamiento emancipador de tipos, métodos, técnicas e instrumentos investigativos diversos, pero incluyentes. La innovación es un campo de batalla de supremacía incorruptible de problemas por omisión o acción, el problema y la presión se transforman en la materia prima de la innovación; y la experiencia es la brújula conducente que apoyada con las estrategias adecuadas minimiza la resistencia y enajenación de cambio. Este cambio solo puede alcanzarse si los elementos de esta planificación educativa se comprometen a condicionar sus estructuras cognitivas y metacognitivas en una cultura de la innovación con filtros fundamentales de cambio radical con determinismo de transformación programática erradicando la concepción reduccionista del pensamiento simplista por el pensamiento complejo, impulsado por la incertidumbre, la innovación confrontada en el espíritu intuitivo con 
soluciones de problemas y la toma de decisiones educativas vigentes en la enseñanza digital.

La virtualidad tiene sus ventajas y desventajas entre la relación de profesores, estudiantes y el desarrollo de los contenidos académicos, que en materia de turismo aumenta la brecha en el desconocimiento práctico de procesos comerciales en esta área de productividad. Relacionar la teoría con la práctica es compleja por la limitada participación directa de los involucrados: agentes internos vs. agentes externos, esto responde a las escasas capacitaciones de la población en general. Los procesos de capacitación turística son cruciales para fomentar el empoderamiento local capaz de ayudar a las comunidades a beneficiarse de las oportunidades que el desarrollo turístico puede ofrecer (Aref et al., 2009; Laverack \& Thangphet, 2009; Moscardo, 2008a). Los procesos conducentes a la capacitación humana en las comunidades apenas han sido objeto de estudio en la literatura turística (Giampiccoli et al., 2014; Laverack \& Thangphet, 2009; Moscardo, 2008a). Estos programas incluyen en varios casos cursos de capacitación turística, pero al igual que ocurre con el resto de las líneas de actuación públicas "no obedecen a la idiosincrasia de las comunidades; por lo que únicamente se convierten en apoyos y no en compromisos reales con la población rural de México" (Garduño et al., 2009, p.26).

Moscardo (2008b), asevera "what is required are case studies and systematic evaluations of different methods of enhancing community knowledge of tourism" (pp.7-8). Aref et al. (2011), concluye que la ausencia de capacidades: recursos, conocimientos, habilidades, educación, falta de sentimiento de propiedad con respecto al turismo y el acceso restringido a la toma de decisiones constituyen las principales limitantes al desarrollo de la industria. Los procesos de capacitación son fundamentales para superar dichas barreras y generar los capitales necesarios en la comunidad para afrontar los retos impuestos por una industria ajena y desconocida, como es el turismo. Bennett et al. (2012), desarrollan un marco de análisis considerando siete activos de capital o bienes comunales: el natural, el físico y construido, el financiero, el político, el institucional, el social, el cultural y el humano. En un análisis similar Koutra y Edwards (2012), analizan el capital social, financiero, humano y físico de dos enclaves costeros en Ghana. El objetivo de los procesos de capacitación reside, por tanto, en desarrollar las fortalezas y habilidades locales para conseguir el empoderamiento de sus miembros (Frank \& Smith, 1999).

Este grupo de autores concluyen que las capacidades necesitan constar en una lógica de asimilación en eficiencia de proyecciones concluyentes sobre realidades obstaculizadas por la negligencia y la mediocridad de autoridades conformistas y cómodas, paralelas al pensamiento visceral con déficit en la democracia y la ciudadanía, el lastre de asimetrías de iniquidad conducen al éxito que sufraga costos inmediatos, no impulsa proyectos a mediano ni largo plazo, espejismo de inequidad producida por el racismo estructural de una economía circular acartonada en la limitada integración y participación de los grupos de atención prioritaria que necesitan rescatar su identidad cultural en cada taller turístico tratado en tiempo real sin discriminación y elaborando una descarga de conocimientos tradicionales. 
Las actuaciones de capacitación exógenas tienden a ser puntuales y limitadas tanto en tiempo como en alcance, lo que impide el empoderamiento efectivo de la comunidad (Manyara \& Jones, 2007).

La capacitación turística debe, además, integrarse en los marcos generales de desarrollo comunitario, con visiones más holísticas y respondiendo a necesidades de formación y estímulo de mayor alcance (Moscardo, 2008a). Sin olvidar que toda acción comunitaria, sea cual sea su objetivo, debe ir acompañada de un proceso de capacitación humana (Simmons et al., 2010). Según Manyara y Jones (2007), la capacitación debe, mantener realidades comunitarias sostenibles y de liderazgo en pleno respeto de las políticas estatales. Nadkarni (2008), dilucida sobre la importancia de romper esquemas polarizados de conocimientos superficiales, propone usar tecnologías de creación, retención, devolución, interpretación y tratamiento del conocimiento, y la implementación de recursos educativos en abierto como herramientas para la capacitación turística en regiones periféricas. Por último, resaltar que, para obtener los deseados objetivos de empoderamiento, la formación debe prestar especial atención al fomento de la confianza, la seguridad y la autoestima de todos los miembros de la comunidad (Manyara \& Jones, 2007; Moscardo, 2008b).

La funcionalidad en los talleres participativos turísticos se encuentra en realidades dimensionales de estructuradas en planes, programas y proyectos ya elaborados en un plan nacional de capacitación realizada por el ministerio de turismo, los departamentos de turismo y la misma cámara provincial de turismo apoyados en la academia. Como innovación a los procesos formativos poblacionales se podría incorporar las siguientes fases para talleres participativos turísticos, como se muestra en la tabla 1.

Tabla 1

Fases para talleres participativos turísticos

\begin{tabular}{lll}
\hline Fases & \multicolumn{1}{c}{ Actos } & \multicolumn{1}{c}{ Acciones } \\
\hline 1 & Participación directa & El ser humano eje primordial de desarrollo \\
2 & Cosmovisión tradicional & Sistemas decodificados \\
3 & Orgullo identidad cultural & Rescatar los valores \\
4 & Responsabilidad asociativa & Stakeholders \\
5 & Importancia convivencia turística & Interculturalidad \\
6 & Revalorización de la empresa & Complementariedad de servicios \\
& conjunta & \\
7 & Replica formativa de líderes y & Acompañamiento endógeno \\
& lideresas & \\
\hline
\end{tabular}

Las comunidades tienen objetos de estudio investigativo; la convivencia y la asociatividad, entonces la capacidad de respuesta está en la simetría de contenidos en confortación de los contextos culturales de los participantes. 
Fases de desarrollo: Fase uno: Participación directa. - El ser humano es el eje primordial de desarrollo es oportuno colocarlo como participante VIP del proceso, considerando dos cosas la toma de decisiones y la solución de problemas, conspirando a entender sus necesidades y metas a futuro. Fase dos: Cosmovisión tradicional. - Estos sistemas decodificados de creencias filosóficas, políticas y otras, propician la riqueza cultural de las comunidades, la puesta en valor al compás del aprovechamiento turístico. Fase tres: Orgullo identidad cultural. - Rescatar los valores y la misma simbología embrionadas en las entrañas mismas la población, este sentido de pertinencia identifica conclaves simultáneos de convivencia mística comunitaria. Fase cuatro: Responsabilidad asociativa. - Es una herramienta utilizada por la pequeña y mediana empresa para enfrentar la globalización. El acondicionamiento de elaboración, organización y comercialización de productos turísticos en base a una cadena de valores, el apoyo de los stakeholders, en mancomunidad genera una ventaja competitiva en la región. Fase cinco: Convivencia turística. - La interrelación entre la comunidad y los visitantes en respeto de las manifestaciones de vida diaria es una perspectiva intercultural previo valoración de sus patrimonios. Fase seis: Revalorización de la empresa conjunta. - Acondicionar las capacidades, iniciativas microempresariales familiares es fundamental para la complementariedad de bienes, servicios, productos y procesos turísticos regionales. Fase siete: Replica formativa de líderes y lideresas. - Conducir a los participantes hacia la compenetración de contenidos entre el resto de la comunidad, ayuda a internalizar lo teórico con lo práctico en construcción comunitaria de los aprendizajes en ejecución y acompañamiento permanente de capacidades.

\section{Metodología}

Esta investigación enfatizó el trabajo de gabinete como primicia de información, la revisión bibliográfica fue fecunda al igual que las experiencias tratando con diferentes pueblos originarios de algunas regiones del país. La constitución antropológica de las comunidades prevalece en su comportamiento y manifestaciones interculturales. La encuesta aplicada a 100 personas de los tres cantones: Babahoyo, Ventanas y Quinsaloma, realizó una interfaz de preferencias para asesoramiento turístico en las comunidades que vincula la participación de los gobiernos autónomos descentralizados municipales y parroquiales, derivando a los recintos como base de corresponsabilidad temeraria frente a la elaboración de talleres participativos turísticos con innovación metodológica educativa para organizaciones campesinas. Se trabajo con indicadores aplicados en zonas rurales de México y por las particularidades de nuestro país y región se construyeron otros indicadores de mayor peso sobre la actividad asociativa y la convivencia como carta de presentación poblacional, en el marco de corresponder a la amabilidad en vínculo comunitario hacia los visitantes. No se encontraron trabajos sustantivos ni significativos sobre metodología de trabajo organizativo con la etnia montuvia. Este trabajo direccionó el comportamiento psicológico turístico hacia la descolonización mental de los miembros comunitarios, en aprovechamiento racional de los recursos que poseen. Se aplicó la técnica de la observación, lista de cotejo para viralizar estructuras cognitivas comunicacionales y procesos dialógicos horizontales sobre servicios turísticos. 


\section{Resultados}

La descripción investigativa tiene carácter cuantitativo, los beneficiarios de los diferentes talleres participativos turísticos mencionan que existen realidades contextuales diferentes en algunos cantones de la provincia de Los Ríos, que serían subsanados con la utilización de material actual, real y de fácil contraste comparativo. La lectoescritura debería formar parte crucial en los talleres participativos turísticos para replicar emprendimientos exitosos en otras regiones, que permitan ser acoplados en el territorial provincial, deben existir indicadores de exigencias de cumplimiento y asistencia, desde el trabajo metodológico motivacional que involucre necesariamente a todos los entes encargados de esta actividad, en particular a la universidad que brinda su talento humano entre profesores y estudiantes hacia el protagonismo de la población.

\section{Tablas 2}

Innovación educativa y talleres participativos turísticos

\begin{tabular}{|c|c|c|c|}
\hline $\begin{array}{c}\text { Indicadores } \\
\text { dominantes Innovación }\end{array}$ & $\begin{array}{c}\text { Indicadores } \\
\text { dominantes Talleres } \\
\text { participativos }\end{array}$ & $\begin{array}{c}\text { Porcentaje de cambio } \\
\text { paradigmático }\end{array}$ & $\begin{array}{c}\text { Acciones } \\
\text { procedimentales en } \\
\text { beneficio de la } \\
\text { creatividad e innovación }\end{array}$ \\
\hline Trabajo autónomo & $\begin{array}{l}\text { Realidad turística } \\
\text { superflua }\end{array}$ & $81 . \%$ & $\begin{array}{l}\text { Clases reflexivas con } \\
\text { contextos reales }\end{array}$ \\
\hline Priorizar problemas & $\begin{array}{l}\text { Analogía de contextos } \\
\text { turísticos }\end{array}$ & $51.7 \%$ & $\begin{array}{l}\text { Lectoescritura reflexiva } \\
\text { y crítica }\end{array}$ \\
\hline $\begin{array}{l}\text { Seguimiento a } \\
\text { propuestas innovadoras } \\
\text { educativas }\end{array}$ & Exigencias del taller & $30.8 \%$ & Investigaciones \\
\hline
\end{tabular}

\section{Conclusiones}

- Los talleres participativos turísticos brindan nuevas alternativas de desarrollo poblacional, las estrategias generadas en estos eventos permiten innovar en emprendimientos complementarios que requieren de adaptaciones curriculares entre los beneficiarios, propiciando actividades productivas y comerciales a corto, mediano y largo plazo.

- El área de emplazamiento turístico vincula a grupos de atención prioritaria a través del asesoramiento entre instituciones estatales y privadas que impulsan el trabajo mancomunado en áreas rurales campesinas apoyado por el trabajo investigación acción poblacional que busca ejecutar talleres participativos turísticos permanentes y manteniendo el correspondiente acompañamiento por las instituciones de turno. 
- Los municipios verifican que las necesidades de capacitación estén enmarcadas en las iniciativas de planificación y desarrollo de país, que el aprovechamiento racional de los recursos naturales y culturales de la provincia se dibuje en respuesta a la conservación y protección de elementos constitutivos en la capacitación (inventario turístico), contribuyendo al bien común de la población involucrada.

- Los líderes comunitarios forman parte irrelevante e invariable en la planificación de este tipo de eventos que contrastan con la negligencia dirigencial y con los gestores administrativos intermedios, sobresaliendo en estas convocatorias los jóvenes y las mujeres, protagonistas de minimizar la migración hacia las urbes poniendo en riesgo su físico y su identidad

\section{Referencias bibliográficas}

Aref, F., Redzuan, M. \& Gill, S. S. (2009). Community skill \& knowledge for tourism development. European Journal of Social Sciences, 8(4), 665-671.

Aref, F., Redzuan, M. \& Gill, S. S. (2011). Dimensions of community capacity building: A review of its implications in tourism development. Journal of American Science, 6(1), 172-180.

Bennett, N., Lemelin, R. H., Koster, R. \& Budke, I. (2012). A capital assets framework for appraising and building capacity for tourism development in aboriginal protected area gateway communities. Tourism Management, 33(4), 752-766. Doi: 10.1016/j.tourman.2011.08.009

Epper, R., Honda \& Tony. Bates. (2004): Enseñar al profesorado cómo usar la tecnología. Buenas prácticas de instituciones líderes, Editorial UOC, Barcelona. University of New Mexico Press.

De Pablos, Pons, J., Jiménez, R. \& Cortés, C. (2007a): «Buenas prácticas con TIC, apoyadas en las políticas educativas: claves conceptuales y derivaciones para la formación en competencias», Revista L.A. de Tecnología Educativa, vol. 6, n.2, Málaga, pp. 15-28.

De Pablos, Pons, J. \& González, T. (2007b): «Políticas e innovación educativas apoyadas en las TIC. Sus desarrollos en el ámbito autonómico», ponencia, II Jornada Internacional sobre Políticas Educativas para la Sociedad del Conocimiento, enero, Morán de la Frontera, Sevilla. D, C (1985): De la animación pedagógica a la investigación-acción:

Frank, F. \& Smith, A. (1999). The community development handbook: A tool to build community capacity. Ottawa, ON: Human Resources Development Canada.

García, H., Trejo, S. \& Licona D. (2012): Incubando proyectos educativos. Los agentes de cambio politécnicos y prácticas innovadoras, Instituto Politécnico Nacional (IPN), México D. F. 
García, V. \& Valcárcel, A. (2007): «Estrategias para una innovación educativa mediante las TIC», Revista L.A. de Tecnología Educativa, vol. 1, n. 2, Málaga, pp. 41-50.

Garduño Mendoza, M., Guzmán Hernández, C. \& Zizumbo Villarreal, L. (2009). Turismo rural: Participación de las comunidades y programas federales. El Periplo Sustentable, 17, 5-30.

Giampiccoli, A., Jugmohan, S. \& Mtapuri, O. (2014). International cooperation, community-based tourism and capacity building: Results from a Mpondoland village in South Africa. Mediterranean Journal of Social Sciences, 5(23), 657-667.

Havelock, R. G. \& Huberman, A. M. (1977). Solving educational problems: the theory and reality of innovation in developing countries. Suiza: UNESCO

Koutra, C. \& Edwards, J. (2012). Capacity building through socially responsible tourism development: A Ghanaian case study. Journal of Travel Research, 51(6), 779-792.

Laverack, G. \& Thangphet, S. (2009). Building community capacity for locally managed ecotourism in northern Thailand. Community Development Journal, 44(2), 172185. doi:10.1093/cdj/bsm058

Manyara, G., \& Jones, E. (2007). Community-based tourism enterprises development in Kenya: An exploration of their potential as avenues of poverty reduction. Journal of sustainable tourism, 15(6), 628-644.

Moscardo, G. (2008a). Innovación turística sostenible: desafiando los supuestos básicos. Investigación en turismo y hostelería, 8 (1), 4-13.

Moscardo, G. M. (2008b). Community capacity building: An emerging challenge for tourism development. In G. M. Moscardo (Ed.), Building community capacity for tourism development

Nadkarni, S. (2008). Knowledge creation, retention exchange, devolution, interpretation and treatment (K-CREDIT) as an economic growth driver in pro-poor tourism. Current Issues in Tourism, 11(5), 456-472.

Palomo, Rafael. Ruiz, J. \& Sánchez, J. (2006): Las TIC como agentes de innovación educativa, Consejería de la Junta de Educación de Andalucía, Sevilla.

Salinas, Jesús. (2004): «Los recursos didácticos y la innovación educativa», Comunicación y Pedagogía, N.200, Madrid, pp. 36-39.

Simmons, B., Bushell, R. \& Scott, J. (2010). Fostering responsible tourism business practices through collaborative capacity-building. In J. Sarkis, J. J. Cordeiro \& D. V. Brust (Eds.), Facilitating sustainable innovation through collaboration: A multi-stakeholder perspective (pp. 185-201) doi:10.1007/978-90-481-3159-4_10 


\section{PARA CITAR EL ARTÍCULO INDEXADO.}

Rivera García, C., Arellano Valencia, D., \& Reyes Indio, X. N. (2021). La innovación educativa y la vinculación con talleres participativos turísticos en la provincia de los Ríos. AlfaPublicaciones, 3(4), 133-142. https://doi.org/10.33262/ap.v3i4.134

\section{Ciencia}

El artículo que se publica es de exclusiva responsabilidad de los autores y no necesariamente reflejan el pensamiento de la Revista Alfa Publicaciones.

El artículo queda en propiedad de la revista y, por tanto, su publicación parcial y/o total en otro medio tiene que ser autorizado por el director de la Revista Alfa Publicaciones.
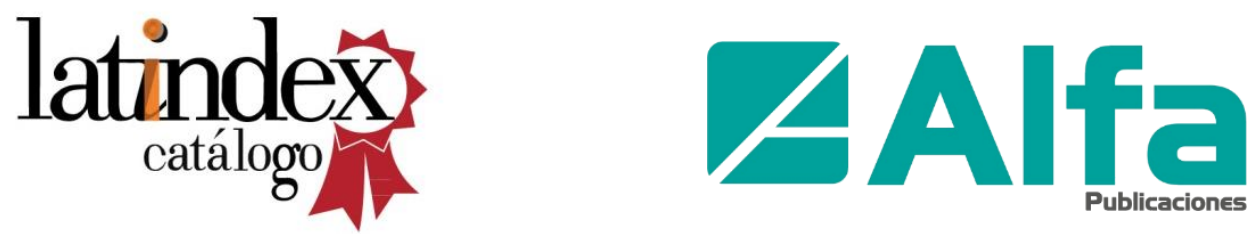